\title{
Motion estimation and compensation in the redundant-wavelet domain using triangle meshes
}

\author{
Suxia Cui ${ }^{\mathrm{a}}$, Yonghui Wang ${ }^{\mathrm{a}}$, James E. Fowler ${ }^{\mathrm{b}, *}$ \\ ${ }^{a}$ Department of Engineering Technology, Prairie View A\&M University, Prairie View, TX 77446, USA \\ ${ }^{\mathrm{b}}$ Department of Electrical and Computer Engineering, GeoResources Institute (GRI), Mississippi State University, Mississippi State, \\ MS 39762, USA
}

Received 9 September 2004; received in revised form 15 March 2006; accepted 16 March 2006

\begin{abstract}
In this paper, a technique is presented that incorporates an irregular triangle mesh into wavelet-domain motion estimation and compensation using a shift-invariant redundant-wavelet transform. The main contribution of this work resides in a demonstration that triangle-mesh motion estimation and compensation can be deployed more effectively in the redundant-wavelet domain thanks to a simple correlation operator that is robust to the prediction residual, a noise-like signal that hinders spatial-domain gradient-based efforts to locate image edges which persist from frame to frame. In the proposed technique, motion compensation takes place through an affine transform mapping triangles from one frame to the next, while the motion-compensated residual is downsampled to a non-redundant form which is then coded using any wavelet-based still-image coder. Experimental results indicate that the proposed technique significantly outperforms not only the usual spatial-domain approach to mesh-based motion estimation and compensation, but also the emerging wavelet-based coders that employ traditional block-based structures, particularly for sequences with fast or complex motion.
\end{abstract}

(C) 2006 Elsevier B.V. All rights reserved.

Keywords: Motion estimation; Motion compensation; Redundant-wavelet transform; Mesh-based motion estimation; Shift-invariant wavelet transform

\section{Introduction}

Block-based motion estimation (ME) and motion compensation (MC) followed by a discrete cosine transform (DCT) is widely employed in modern video-compression systems and an integral part of

\footnotetext{
${ }^{*}$ Corresponding author. Tel.: + 16623252578 .

E-mail addresses: sucui@pvamu.edu (S. Cui), yowang@pvamu.edu (Y. Wang), fowler@ece.msstate.edu (J.E. Fowler).
}

standards such as H.263, MPEG-2, and MPEG-4. However, given the promising performance of wavelet-based still-image compression algorithms such as SPIHT [26] and JPEG-2000, there has been recent interest in deploying $\mathrm{ME} / \mathrm{MC}$ within such algorithms to produce wavelet-based video coders. On another front, there have been a variety of proposals for employing geometries more general than square blocks to drive $\mathrm{ME} / \mathrm{MC}$. In this paper, we combine both of these recent developments. Specifically, we use $\mathrm{ME} / \mathrm{MC}$ based on triangle 
meshes in the wavelet domain and find that this strategy can be significantly more effective than both the usual spatial-domain approach to trianglemesh $\mathrm{ME} / \mathrm{MC}$ as well as the other wavelet-based coders that employ traditional block-based ME/MC structures.

The fact that the critically sampled discrete wavelet transform (DWT) used ubiquitously in image-compression efforts is shift variant has long hindered the $\mathrm{ME} / \mathrm{MC}$ process in the wavelet domain. However, in [25], it was recognized that difficulties associated with the shift variance of traditional DWTs could be overcome by choosing instead to perform $\mathrm{ME} / \mathrm{MC}$ in the domain of a redundant transform. In essence, the redundant DWT (RDWT) [12,17,29] removes the downsampling operation from the traditional DWT to ensure shift invariance at the cost of a redundant, or overcomplete, representation.

Whereas the RDWT-based ME/MC of [25] is based on the square-block structure long a staple of traditional video coding, the approach we propose in this paper drives RDWT-domain ME/MC with an irregular triangle mesh. The motivation for mesh-based $\mathrm{ME} / \mathrm{MC}$ is that a mesh structure can oftentimes better match the motion of objects in video. In mesh-based approaches, such as trianglemesh ME/MC (e.g., [13]), the regions are sized and shaped accordingly to the local level of detail in the image. Specifically, in triangle-mesh ME/MC, triangle vertices, or control points, are selected to track those salient image features, such as edges of objects, which tend to persist from frame to frame.

As the main contribution of this paper, we demonstrate that triangle-mesh $\mathrm{ME} / \mathrm{MC}$ can be deployed more effectively in the RDWT domain than in the spatial domain. Specifically, we argue that the motion of signal features can be modeled as a simple translation with added residual noise not captured by the translational motion. Since edge detection in the spatial domain typically relies on some form of gradient detection, and since the gradient is exceedingly sensitive to noise, the residual noise signal in the $\mathrm{ME} / \mathrm{MC}$ process hinders accurate identification of those edges that persist from frame to frame. On the other hand, we show that a simple correlation operator can be easily employed in the RDWT domain so that interference from the residual noise is rejected, and accurate binding of control points to persistent object edges is achieved. To support this argument, we present empirical results that demonstrate that the combi- nation of triangle meshes with RDWT-domain ME/ $\mathrm{MC}$, which we name redundant-wavelet trianglemesh (RWTM) ME/MC, significantly outperforms either approach employed in isolation.

We emphasize that, throughout this paper, we focus merely on the $\mathrm{ME} / \mathrm{MC}$ process rather than attempt to develop a full-featured, state-of-the-art video-coding system. In fact, most recent interest in wavelet-based video coding has migrated away from the traditional hybrid MC-feedback architecture considered here in favor of motion-compensated temporal filtering (MCTF) in order to provide full fidelity, spatial, and temporal scalability. Recent MCTF-based video coders have employed the RDWT (e.g., [33,2,22]), while others have used meshes (e.g., [27,28]). Indeed, our recent work $[31,30]$ has focused on combining uniform meshes and the RDWT within the MCTF framework and has produced a scalable coder with state-of-the-art rate-distortion performance. It is in this arena of mesh-based MCTF that we expect that the current work will have the most impact, and we are currently investigating the deployment of the correlation-based RWTM proposed here within the MCTF architecture of $[31,30]$.

The remainder of this paper is organized as follows. In the next section, we overview the RDWT and prior techniques for $\mathrm{ME} / \mathrm{MC}$ in the RDWT domain. Then, in Section 3, we argue for the superiority of the RDWT domain for triangle-mesh $\mathrm{ME} / \mathrm{MC}$, discussing both the inaccuracy of spatialdomain gradient-based operators to residual noise as well as the robustness of RDWT-based correlators to the same. We follow with a detailed overview of the RWTM ME/MC technique in Section 4, while experimental results comparing RWTM to other $\mathrm{ME} / \mathrm{MC}$ techniques are presented in Section 5. Finally, we make some concluding remarks in Section 6.

\section{ME/MC and the RDWT}

The RDWT can be considered to be an approximation to the continuous wavelet transform that removes the downsampling operation from the traditional critically sampled DWT to produce an overcomplete representation. The shift-variant characteristic of the DWT arises from its use of downsampling, while the RDWT is shift invariant since the spatial sampling rate is fixed across scale. The RDWT has been given several appellations over the years, including the "undecimated DWT," 
the "overcomplete DWT," "discrete wavelet frames," and the algorithme à trous. The reader is referred to $[12,17,29,15,11]$ for greater detail on the RDWT, its implementations, and its relation to the critically sampled DWT.

There are a several equivalent ways to implement the RDWT, and several ways to represent the resulting overcomplete set of coefficients. The most popular coefficient-representation scheme employed in RDWT-based video coders is that of a coefficient tree. This tree representation is created by employing filtering and downsampling as in the usual critically sampled DWT; however, all sets, or phases, of downsampled coefficients are retained and arranged in a tree-like fashion. It is straightforward to see that each path from root to leaf in the RDWT tree constitutes a distinct critically sampled DWT (see [15]). The RDWT was originally formulated, however, as the the algorithme à trous implementation [12,17]. In this implementation, decimation following wavelet filtering is eliminated, and, for each successive scale of decomposition, the filter sequences themselves are upsampled, creating "holes" of zeros between non-zero filter taps. As a result, the size of each subband resulting from an RDWT decomposition is exactly the same as that of the input signal, as is illustrated for a $2 \mathrm{D}$ image in Fig. 1. The advantage of this spatially coherent representation is that each RDWT coefficient is located within its subband in its spatially correct position. As illustrated in Fig. 2, by appropriately subsampling each subband of an RDWT, one can produce exactly the samecoefficients as does a criticially sampled DWT applied to the same input signal (see [15]). Below, we use this spatially coherent representation as the basis for a correlation operator designed to guide the placement of a triangle mesh for $\mathrm{ME} / \mathrm{MC}$.

The majority of prior work concerning RDWTbased video coding originates in the work of Park and Kim [25], in which the system shown in Fig. 3 was proposed. In essence, the system of Fig. 3 works as follows. An input frame is decomposed with a critically sampled DWT and partitioned into blocks, wherein each $N \times N$ block is composed of the coefficients from each subband that correspond spatially to a particular $N \times N$ block in the original image. A full-search block-matching algorithm is then used to compute motion vectors for each wavelet-domain block; the system uses as the reference for this search an RDWT decomposition of the previous reconstructed frame. Since these reconstructed RDWT coefficients are arranged in the tree representation as described above, the motion-estimation procedure of this system amounts to identifying, for each block of the current frame, a particular critically sampled DWT in the tree (a root-to-leaf path), and a displacement within that DWT. Transmission of a single motion vector per block suffices to convey all

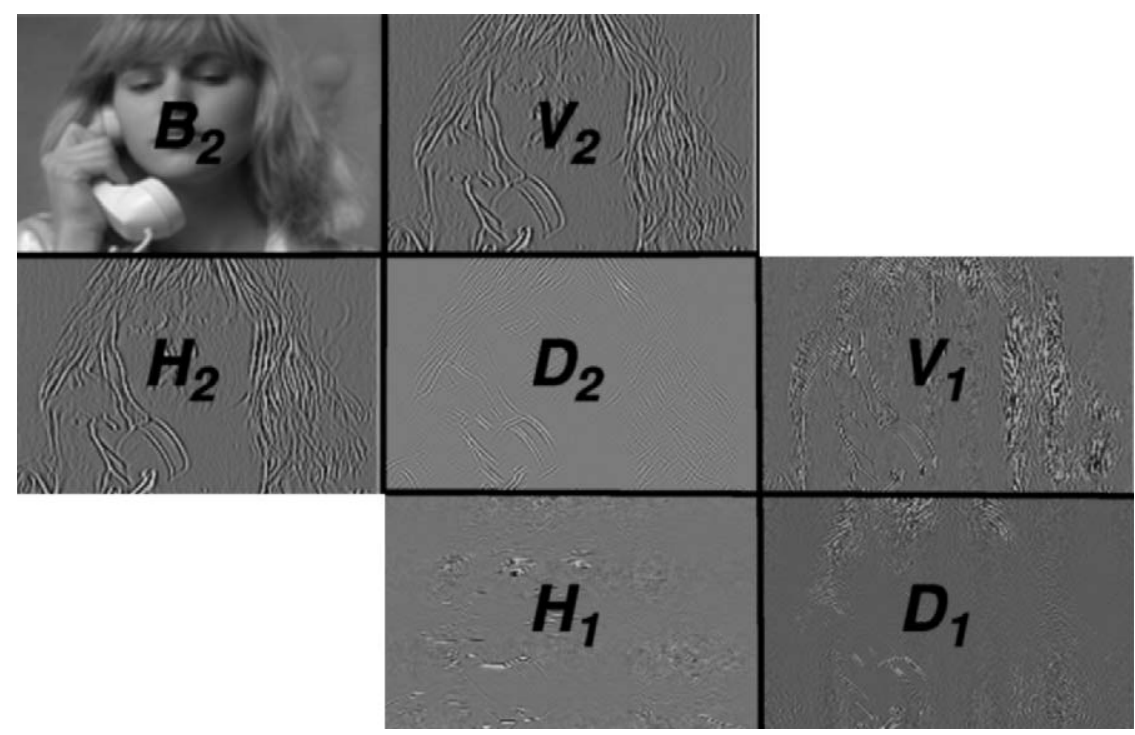

Fig. 1. Spatially coherent representation of a two-scale RDWT of a 2D image. Coefficients retain their correct spatial location within each subband, and each subband is the same size as the original image. $B_{j}, H_{j}, V_{j}$, and $D_{j}$ denote the baseband, horizontal, vertical, and diagonal subbands, respectively, at scale $j$. 

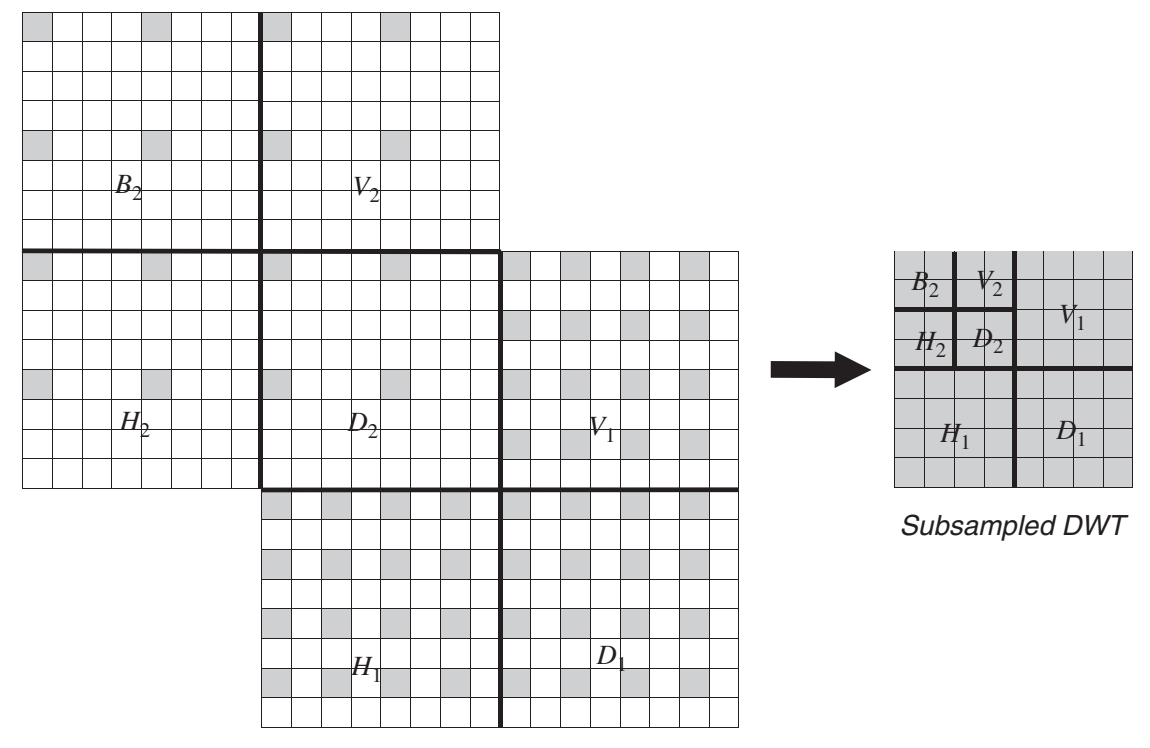

Subsampled DWT

RDWT

Fig. 2. Subsampling of a 2 -scale $2 \mathrm{D}$ RDWT to recover a critically sampled DWT. A $J$-scale $2 \mathrm{D}$ RDWT contains $4^{J}$ distinct critically sampled DWTs [15].

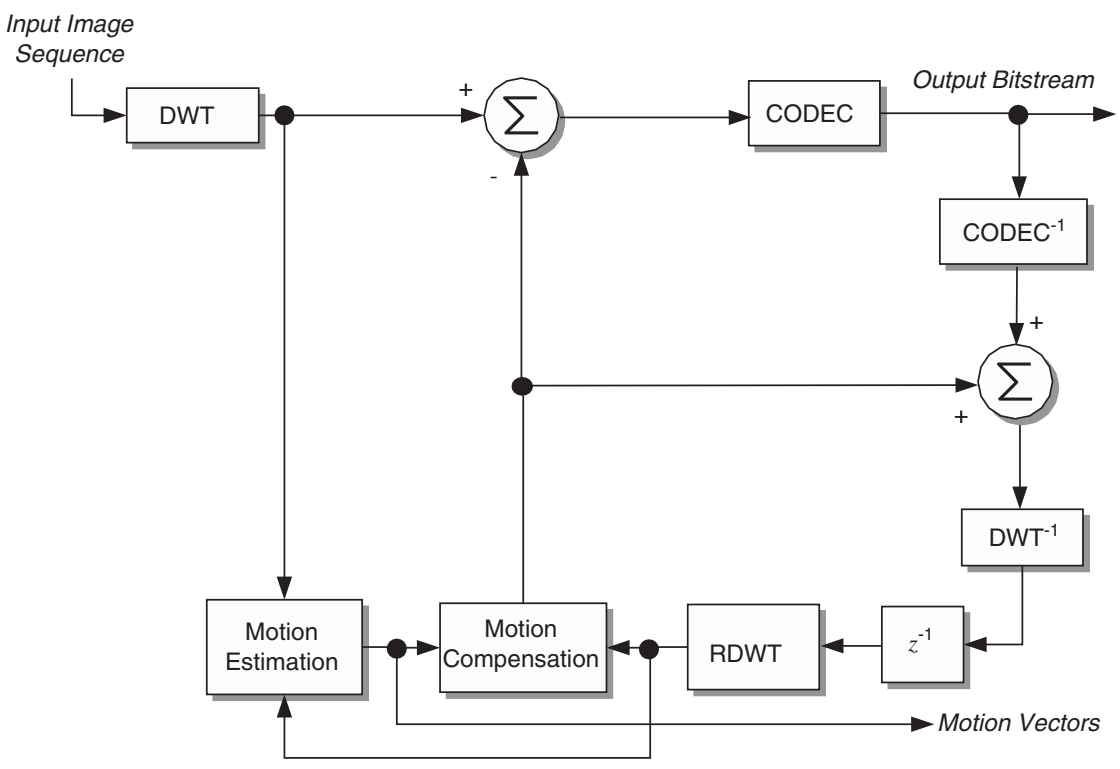

Fig. 3. The RDWT-based video coder of [25]. $z^{-1}=$ frame delay, CODEC is any still-image coder operating in the critically sampled DWT domain.

of this motion information to the decoder. Subsequent work has offered refinements to the system depicted in Fig. 3, such as the deriving of motion vectors for each subband [20,3], or resolution [24], independently; sub-pixel accuracy ME [23]; resolution-scalable coding [24,3,4]; and multihypothesis prediction via the redundant RDWT phases $[15,11]$.
Most of these prior approaches to RDWTbased video exploit the redundancy of the RDWT simply for its shift invariance. Below, we argue that the redundancy of the RDWT can also facilitate the edge-detection process that underlies the deploying of triangle meshes for $\mathrm{ME} / \mathrm{MC}$. 


\section{Motion estimation with triangle meshes}

\subsection{Edge detection and noise}

In ME based on triangle meshes (e.g., [13]), one tracks object motion by mapping triangles in one frame into another frame. This mapping is accomplished by tracking the motion of control pointsthe triangle vertices-between frames. Thus, the choosing of proper control points is crucial to the accuracy of triangle-mesh ME. Although it is possible to deploy meshes independently of scene content, ideally, one would like control points to track salient image features which persist from frame to frame. Since edges of objects are likely to be among the most persistent features in a scene, the first step to creating a triangle mesh for $\mathrm{ME}$ typically involves the application of some edgedetection operator.

The most common approach to edge detection in the spatial domain is to identify maxima in the magnitude of a gradient operator applied to the image. However, because of the exceedingly noisesensitive nature of the gradient, spatial-domain edge detectors based on the gradient can become quite inaccurate in the presence of noise [19].

On the other hand, the redundancy of the RDWT facilitates the identification of salient features in an image, especially image edges, since a simple correlation operation can easily accomplish edge identification [32]. Specifically, the direct multiplication of the RDWT coefficients at adjacent scales distinguishes important features from the background due to the fact that wavelet-coefficient magnitudes are correlated across scales. In addition, since uncorrelated noise added in the spatial domain remains uncorrelated in the RDWT domain, the correlation operator tends to reject noise and identify only true signal edges. We note that feature correlation and noise decorrelation is well-known to exist in the usual critically sampled DWT also; however, the changing spatial sampling rate of the critically sampled DWT makes the calculation of an explicit correlation mask across scales much more difficult [32].

Fig. 4 illustrates the superior edge-detection performance of an RDWT-based correlation mask as compared to traditional gradient-based edge detection in the spatial domain. We see that the RDWT correlation-based detector provides much greater edge-detection accuracy in the presence of random noise. Below, we argue that this observa-

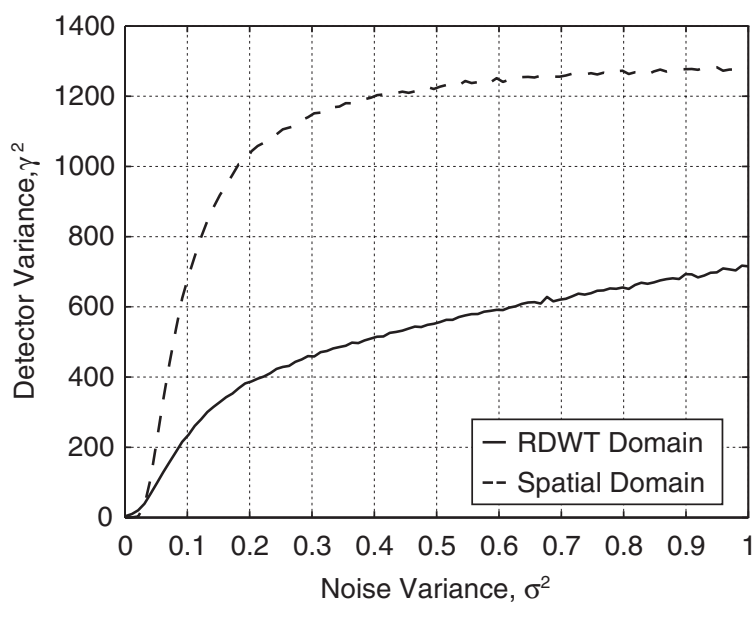

Fig. 4. Accuracy of edge detection in the presence of noise. $\sigma^{2}$ is the variance of zero-mean white Gaussian noise added to an ideal unit-step edge. The spatial-domain approach identifies maxima in a gradient operator with impulse response $[-1,0,1]$, essentially a 1D equivalent of the well-known Sobel edge detector [19]. The RDWT-domain approach consists of identifying maxima in the correlation mask as calculated by multiplying together all subbands of a 5-scale RDWT in the style of [32]. Accuracy is measured as the variance, $\gamma^{2}$, of the detector output over 100,000 trials.

tion implies that triangle-mesh $\mathrm{ME} / \mathrm{MC}$ can be carried out more effectively in the RDWT domain than in the spatial domain.

\subsection{Implications for motion estimation}

For accurate ME/MC with meshes, we want to anchor mesh control points to signal features that persist in time from frame to frame. The above discussion suggests that we can more accurately identify persistent edge features in the RDWT domain than in the spatial domain through the following argument. A simple model [8] of the MC process in the spatial domain is

$I^{\mathrm{cur}}(x, y)=I^{\mathrm{ref}}\left(x-\Delta_{x}, y-\Delta_{y}\right)+E(x, y)$,

where $I^{\text {cur }}$ is the current frame, $I^{\text {ref }}$ is the reference frame, $\left(\Delta_{x}, \Delta_{y}\right)$ is the motion vector for pixel $(x, y)$, and $E(x, y)$ is the prediction residual resulting from the $\mathrm{ME} / \mathrm{MC}$ process. It has been established that the more accurate the ME estimation process is, the more the prediction residual $E$ appears as uncorrelated "noise," i.e., a spatially unstructured signal with approximately flat power spectrum [16,9]. Thus, (1) describes the motion of signal features such as edges as a simple translation plus additive 
residual noise. (1) also implies that the signal features present in the current frame $I^{\text {cur }}$ can be detected in the reference frame $I^{\text {ref }}$ (which is $I^{\text {cur }}-E$ ) provided that the feature detector used is robust to the additive residual noise $E$, that is, provided that the feature detector is insensitive to noise $E$ in $I^{\text {cur }}-E$.

By this reasoning, Fig. 4 suggests that the RDWT domain provides a more suitable setting for $\mathrm{ME} /$ $\mathrm{MC}$ with triangle meshes than the spatial domain since the selection of persistent edge features can be done more accurately in the RDWT domain due to the resilience of the correlation mask to added noise. Specifically, as ME becomes more accurate, the residual $E$ becomes more noise-like, and Fig. 4 becomes increasingly relevant. In the spatial domain, the noisy character of the residual $E$ increasingly interferes with the accurate identification of persistent edge features in $I^{\text {ref }}$, thereby discouraging further improvement in ME accuracy. On the other hand, as the residual starts to increasingly resemble uncorrelated noise, the correlation mask in the RDWT domain in fact becomes more effective at rejecting the residual from the edge-detection process, thereby encouraging even greater edgeidentification accuracy. That is to say, as the residual looks increasingly like noise, it is more likely that an edge determined to be salient in $I^{\text {ref }}$ actually corresponds a prominent edge feature in $I^{\text {cur }}$; i.e., it is more likely that a signal feature which is actually persisting across the two frames has been identified. Below, we use this observation as the basis for the proposed RWTM approach to ME/ MC which uses an RDWT-based correlation mask to guide the deployment of a triangle mesh in the redundant-wavelet domain.

\section{The RWTM technique for ME/MC}

The RWTM approach we propose here ${ }^{1}$ departs significantly from the RDWT-based ME/MC architecture originating in [25] and shown in Fig. 3. Specifically, our proposed RWTM adapts the triangle-mesh $\mathrm{ME} / \mathrm{MC}$ technique of [13] to the wavelet domain by replacing the block-based $\mathrm{ME} /$ MC of [25] and similar techniques with trianglemesh ME/MC. This modification necessitates that we employ all phases of an RDWT of the current input frame to estimate motion rather than a single

\footnotetext{
${ }^{1} \mathrm{We}$ note that a preliminary description of RWTM was presented in [10].
}

critically sampled DWT of the current frame as was the case in [25].

Our RWTM approach to ME/MC is depicted from the encoding side in Fig. 5. The input image is first transformed using a RDWT, and control points are identified in the previous reference frame by locating the most salient image edges. The motion of these control points from the reference frame to the current frame is estimated in the RDWT domain, and motion vectors are transmitted to the decoder to allow it to track control-point motion. $\mathrm{MC}$ is accomplished by first using a triangulation algorithm to generate a triangle mesh on the control points in the reference frame and then using affine transformations to predict, subband by subband, triangles in the current frame from triangles in the reference frame. Residing in the RDWT domain, the motion-compensated residual is itself redundant; consequently, it is downsampled before coding. The final encoding step consists of a wavelet-domain still-image coder; for the experiments below, we use SPIHT [26], but any waveletdomain still-image coder would suffice.

At the decoder side, motion of the control points is tracked, and a triangulation in the reference frame identical to that used in the encoder is produced. A reconstructed spatial-domain image is produced by inverting the still-image coding, adding on a subsampled RDWT-domain prediction, and inverting the DWT. Finally, an RDWT operation produces the reference-frame subbands for generating the prediction of the next-frame subbands in the RDWT domain. Below, we explore the various components of our proposed RWTM approach to $\mathrm{ME} / \mathrm{MC}$ in greater detail.

\subsection{Selection of control points}

To create the correlation mask for the reference frame, we multiply the vertical $(V)$, horizontal $(H)$, and diagonal $(D)$ bands together across scales and combine the products; i.e.,

$$
\begin{aligned}
\operatorname{mask}(x, y)= & \left|\prod_{j=J_{0}}^{J_{1}} V_{j}(x, y)\right|+\left|\prod_{j=J_{0}}^{J_{1}} H_{j}(x, y)\right| \\
& +\left|\prod_{j=J_{0}}^{J_{1}} D_{j}(x, y)\right|,
\end{aligned}
$$

where $J_{0}$ and $J_{1}$ are the starting and ending scales, respectively, of the correlation operation. Each of the product terms on the right side of (2) is 


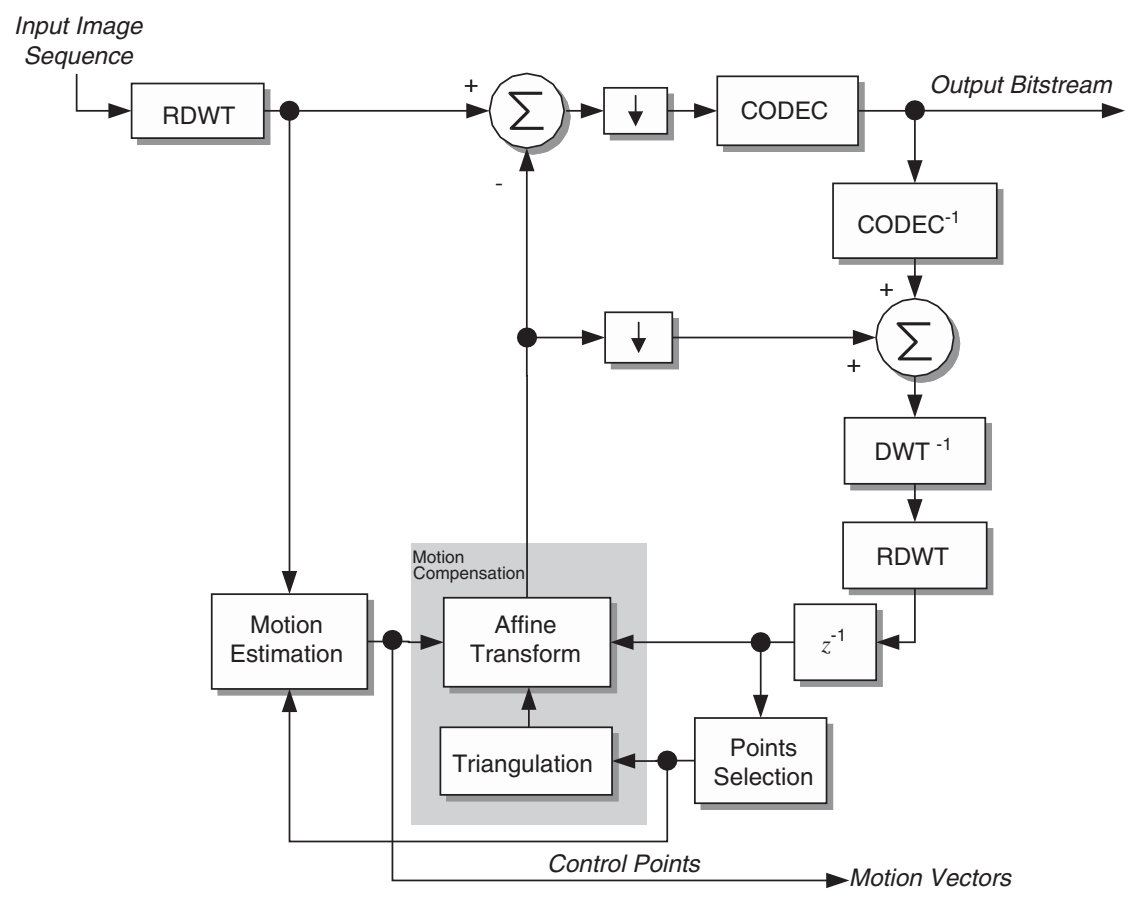

Fig. 5. The RWTM approach to ME/MC. $z^{-1}=$ frame delay, $\downarrow=$ subsampling from RDWT to DWT domains, CODEC is any still-image coder operating in the critically sampled DWT domain.

essentially a $2 \mathrm{D}$ version of the edge-identification process originating in [32]; these terms tend to identify vertical, horizontal, and diagonal edges, respectively. Addition of these product terms thus collects all identified edges into a single correlation mask. We note that calculation of the correlation mask in this manner is possible due to the fact that each RDWT subband is the same size as the original image. Fig. 6 shows the correlation mask for the first frame of the sequence "Susie," where we use the subbands from the two highest-frequency scales in the products above.

To identify control points within the correlation mask, we have devised the following procedure which attempts to place control points on the most salient image edges while ensuring a somewhat uniform spatial spread of the control points across the image. We first label $\alpha$ percent of the locations with the highest mask values as candidate control points. We next divide the mask into $M \times M$ blocks and select at most one candidate control point in each block as a final control point, processing the $M \times M$ blocks in raster scan order. Specifically, in each block, we select a candidate control point that is located a distance of $d_{\min }$ or greater from an already identified final control point; if there are

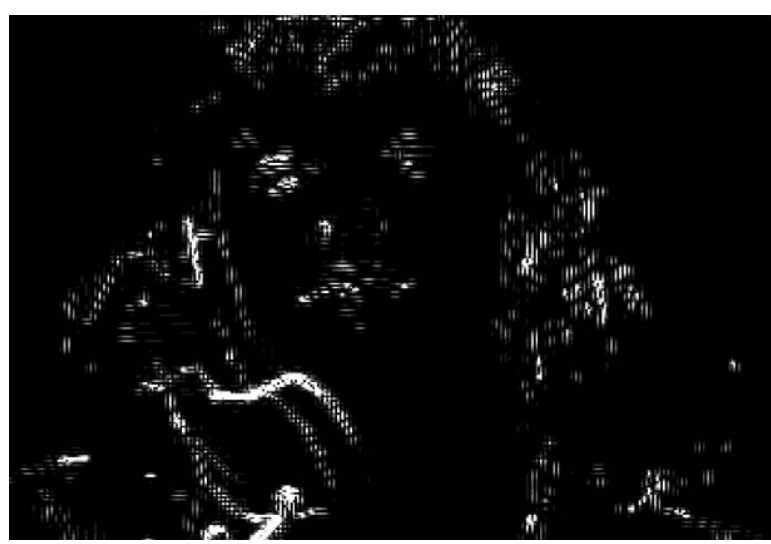

Fig. 6. Correlation mask for the first frame of "Susie".

multiple candidate control points in the current block that satisfy this minimum-distance criterion, we pick the one with the highest mask value. Finally, we add control points equally spaced along the image border to the points chosen via the correlation mask so that the meshed area covers the entire image. These border points always have zero motion vectors and thus are not included in the motion-vector information transmitted by the encoder. 
By enforcing a minimum distance between selected control points, and by selecting the points block by block, we discourage the occurrence of triangles with very acute angles which can lead to numerical problems during $\mathrm{MC}$, as discussed in Section 4.3. Additionally, we also encourage that some control points are devoted to the tracking of some background objects rather than being focused exclusively on the most salient foreground objects.

The critical parameter in our control-point selection process is the $\alpha$ value used to determine candidate control points. A large $\alpha$ will permit a large number of control points to be selected, up to a maximum of one per block, while a relatively small value of $\alpha$ will reduce the number of motion vectors, and, consequently, the proportion of the rate devoted to motion-vector overhead. We have determined empirically that the best $\alpha$ is largely dependent on rate independent of the sequence being coded; by exhaustively evaluating performance over a set of $\alpha$ values to select rates on various video sequences, we have devised the following rate-dependent formula for $\alpha$,

$\alpha= \begin{cases}\xi^{R-0.5} \times 100 \%, & R \leqslant 0.5, \\ 100 \%, & R>0.5,\end{cases}$

where $R$ is rate in bits-per-pixel (bpp), and constant $\xi=1.6269 \times 10^{6}$.

\subsection{Motion estimation}

Each non-border control point identified in the reference frame via the correlation mask has an associated motion vector describing the movement of that control point from the reference frame to the current frame. These motion vectors are obtained by finding the best-matching point in the current frame for each control point in the reference frame. This match is accomplished by calculating the absolute difference of a $B \times B$ block centered at the control point in the reference frame and blocks in a search window about the control-point location in the current frame, similar to the usual blockbased ME process. Our triangle-mesh ME is quite similar to the triangle-mesh ME proposed in [13] in the spatial domain. However, because our ME takes place in the RDWT domain, for a given vector in the search window, we calculate absolute differences for all the subbands at all scales and sum them together to produce a cross-subband, cross-scale distortion, as was proposed in [25] for block-based
ME in the RDWT domain. We choose the vector that minimizes this cross-subband, cross-scale distortion as the motion vector for the current control point.

Specifically, the motion vector, $\left(\Delta_{x}, \Delta_{y}\right)$, for control point $(x, y)$ in the reference frame is the vector in the search window about $(x, y)$ in the current frame that minimizes the mean absolute error (MAE),

$\operatorname{MAE}\left(x, y, \Delta_{x}, \Delta_{y}\right)$

$$
=\frac{1}{B^{2}} \sum_{k=1}^{B} \sum_{l=1}^{B} \mathrm{AE}\left(x+k, y+l, \Delta_{x}, \Delta_{y}\right) .
$$

The absolute error (AE) is

$$
\begin{aligned}
& \operatorname{AE}\left(x, y, \Delta_{x}, \Delta_{y}\right) \\
&=2^{-J}\left|B_{J}^{\mathrm{cur}}\left(x+\Delta_{x}, y+\Delta_{y}\right)-B_{J}^{\mathrm{ref}}(x, y)\right| \\
&+\sum_{j=1}^{J} 2^{-j}\left\{\left|V_{j}^{\mathrm{cur}}\left(x+\Delta_{x}, y+\Delta_{y}\right)-V_{j}^{\mathrm{ref}}(x, y)\right|\right. \\
&+\left|H_{j}^{\mathrm{cur}}\left(x+\Delta_{x}, y+\Delta_{y}\right)-H_{j}^{\mathrm{ref}}(x, y)\right| \\
&\left.+\left|D_{j}^{\mathrm{cur}}\left(x+\Delta_{x}, y+\Delta_{y}\right)-D_{j}^{\mathrm{ref}}(x, y)\right|\right\}
\end{aligned}
$$

where cur and ref denote subbands from the current and reference frames, respectively, and $B_{j}, H_{j}, V_{j}$, and $D_{j}$ are the baseband, horizontal, vertical, and diagonal subbands, respectively, at scale $j$. In the search, motion vectors are chosen from a window of size $W>0$ such that $-W \leqslant \Delta_{x}, \Delta_{y} \leqslant W$.

\subsection{Triangulation and affine transformation}

As in the spatial-domain triangle-mesh $\mathrm{ME} / \mathrm{MC}$ of [13], after the control points are selected in the reference frame, a triangle mesh is computed using Delaunay triangulation. A single triangle mesh is used for all subbands of the RDWT as depicted in Fig. 7; this is possible since each RDWT subband has the same size. MC proceeds by mapping each triangle in the reference frame into the current frame using an affine six-parameter model as described in [1]; this affine mapping is performed for each triangle in each subband separately.

In homogeneous coordinates, affine transforms can represent translation, rotation, and scaling. Consequently, an affine transform can map a point inside one triangle to a point inside another triangle. The affine transform is a 

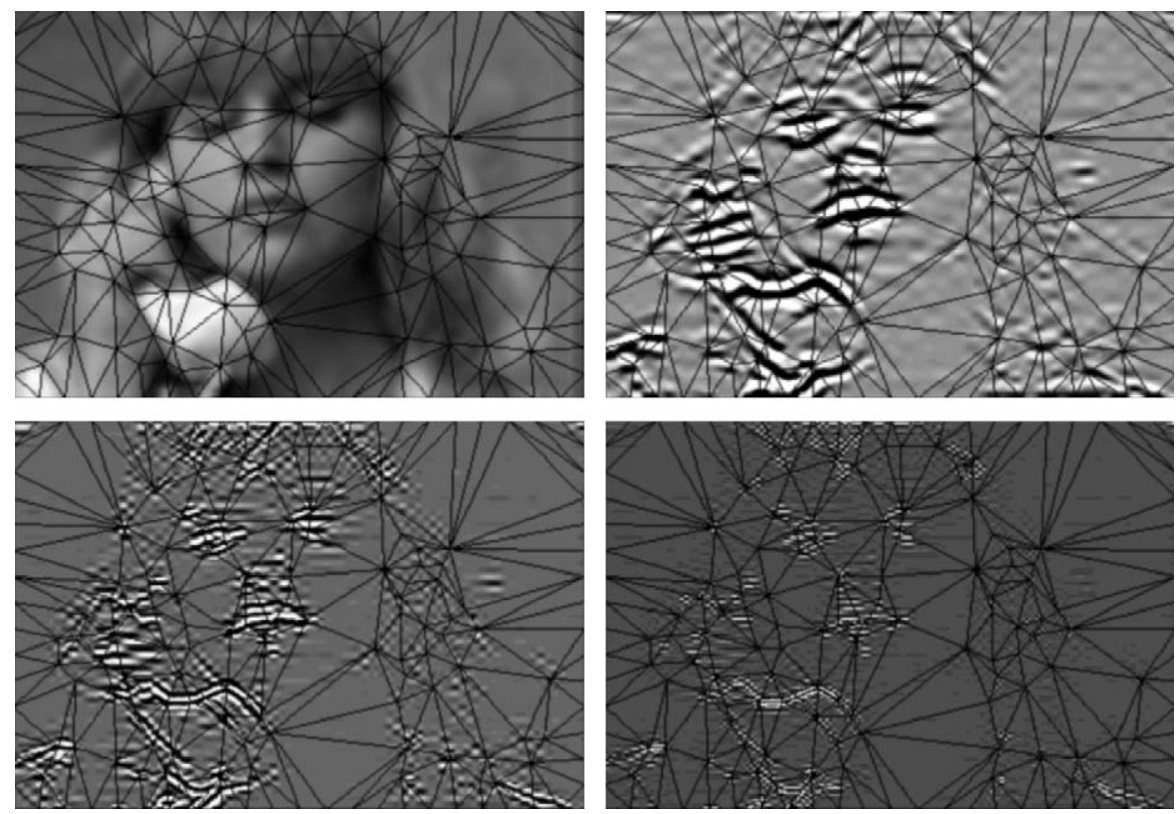

Fig. 7. RDWT subbands and triangle mesh for the first frame of "Susie." Clockwise from upper-left: baseband, $B_{3}$; vertical subband $V_{3}$; subband $V_{1}$; and subband $V_{2}$. A single triangle mesh is applied to all subbands at all orientations and scales, even though only the vertical subbands are shown here.

vector-matrix equation,

$\left[\begin{array}{c}x^{\prime} \\ y^{\prime} \\ 1\end{array}\right]=\left[\begin{array}{ccc}a_{1} & a_{2} & a_{3} \\ b_{1} & b_{2} & b_{3} \\ 0 & 0 & 1\end{array}\right]\left[\begin{array}{l}x \\ y \\ 1\end{array}\right]$,

where $x$ and $y$ are the coordinates of a coefficient in a triangle in the current frame, $x^{\prime}$ and $y^{\prime}$ are the corresponding coordinates in the reference-frame triangle, and $a_{1}, a_{2}, a_{3}, b_{1}, b_{2}$, and $b_{3}$ are the six parameters of an affine transform that is determined for each pair of current- and reference-frame triangles independently. To determine the transform parameters, we evaluate (6) for each of the three vertices of the triangle in the current frame using the known relation between the current- and referenceframe vertices,

$\left[\begin{array}{l}x^{\prime} \\ y^{\prime}\end{array}\right]=\left[\begin{array}{l}x \\ y\end{array}\right]-\left[\begin{array}{l}\Delta_{x} \\ \Delta_{y}\end{array}\right]$,

to yield six equations in six unknowns. Once the parameters of the transform are determined, it is applied to a coefficient location in the current frame to determine the corresponding location in the reference frame, from which a prediction of the coefficient is determined. Bilinear interpolation is employed to calculate predictions for locations that lie off the RDWT-coefficient grid in the reference frame. In order to maximize computational efficiency, the affine transformation is carried out for only those coefficients in the current frame that will survive the subsequent RDWT-to-DWT downsampling operation.

We note that if either triangle has very acute angles such that its three vertices are close to being collinear, then the system of equations producing the affine transformation between the triangles may be ill-conditioned, resulting in possibly inaccurate $\mathrm{MC}$ between the pair of triangles. As a consequence, we devised the procedure for control-point selection described above in Section 4.1 to discourage the formation of such triangles by ensuring a minimum distance between vertices in the reference frame.

Finally, we emphasize that the decoder creates exactly the same triangle mesh in the reference frame as does the encoder, since both the encoder and decoder identify exactly the same control points via identical correlation-mask procedures and produce exactly the same triangles on these points using identical Delaunay triangulation. Thus, transmission of motion vectors describing the movement of each control point of the mesh permits the decoder to not only deduce the mesh for the current frame but also derive the same affine transformation as used by the encoder for each triangle by the process described above. 


\section{Experimental results}

Experimental results use the sequences listed in Table 1. All sequences have CIF resolution $(352 \times 288)$ except "Football," "Susie," and "NYC" which are SIF $(352 \times 240)$. All sequences are 100 frames long except "Susie" (70 frames) and "Foreman" (90 frames). All sequences are grayscale and have a temporal sampling of $30 \mathrm{frames} / \mathrm{s}$ (non-interlaced). The first frame is intra-encoded (I-frame) while all subsequent frames use ME/MC (P-frames). All wavelet transforms (DWT and RDWT) use the the popular $\frac{9}{7}$ biorthogonal filter [7] with symmetric extension and a decomposition of $J=3$ levels. Motion vectors in all systems are coded using the Huffman-based variable-length code for motion-vector data (MVD) of H.261 [18]. All ME/MC methods use integer-pixel accuracy, and all rate figures include motion-vector overhead. The core compression engine in all experiments is the QccPack [14] implementation of SPIHT [26]; since SPIHT produces an embedded coding, each frame of the sequence is coded at exactly the specified target rate.

For our proposed RWTM technique, we calculate the correlation mask of (2) using all scales of decomposition for all sequences; i.e., $J_{1}=J=3$ and $J_{0}=1$. We select control points in the mask using $M \times M$ blocks, ensuring compliance with a minimum distance of $d_{\mathrm{min}}$. For the experiments here, we use $M=16, d_{\min }=8$, and (3) for $\alpha$ for all sequences. To estimate motion of the control points, we use a block of size $B \times B$ centered around the control point in the reference and search in a window of $\pm W$ in the current frame. For the results here, we use $B=17$ and $W=15$ for all sequences.

We compare our proposed RWTM technique to both block- and mesh-based ME/MC in both the spatial and wavelet domains. Specifically, in these results, "Spatial Block" refers to block-based ME/ $\mathrm{MC}$ in the spatial domain followed by a full-frame, critically sampled DWT and SPIHT coding. On the other hand, "RDWT Block" is the technique proposed in [25] which employs block-based ME/ MC to locate DWT blocks in the RDWT domain; afterward, SPIHT coding is applied to the waveletdomain MC residual. Both the Spatial Block and RDWT Block techniques use full-search, blockbased $\mathrm{ME} / \mathrm{MC}$ with fixed-sized blocks of size $16 \times 16$, a search window of size \pm 15 , and integerpixel accuracy. Finally, "Spatial Mesh" is an irregular triangle-mesh $\mathrm{ME} / \mathrm{MC}$ in the spatial domain [13], followed by a full-frame, critically sampled DWT and SPIHT. In this technique, the triangle mesh is deployed in the spatial domain based on edge detection using the well-known Sobel gradient-based operator [19]; otherwise, the aspects of the mesh (selection of control points, controlpoint ME, etc.) are the same as those used for our RWTM technique.

PSNR values averaged over all frames of the sequences are tabulated in Table 1 for a fixed bit rate, while Figs. 8-11 show the rate-distortion performance over a variety of rates for four of the sequences, three with relatively fast or complex motion (Figs. 8-10) and one with slow, simple motion (Fig. 11). The experimental results shown in Table 1 and Figs. 8-11 indicate that our proposed RWTM method outperforms other ME/MC techniques operating in both the spatial and wavelet domains. We see that, for the sequences with fast or complex motion which make up a majority of those listed in Table 1, RWTM outperforms its nearest competitor (RDWT Block) by roughly $0.4-0.8 \mathrm{~dB}$ over wide range of bit rates. RWTM also exhibits a more modest $0.1-0.3 \mathrm{~dB}$ gain over the other

Table 1

Distortion averaged over all frames of the sequence for rate $=0.5 \mathrm{bpp}$

\begin{tabular}{lllll}
\hline & \multicolumn{2}{l}{ PSNR $(\mathrm{dB})$} & & \\
\cline { 2 - 5 } & Spatial Block & Spatial Mesh & RDWT Block \\
\hline Foreman & 37.1 & 37.7 & 38.1 & 33.6 \\
Table-tennis & 32.5 & 33.3 & 31.3 & 37.9 \\
Coastguard & 30.7 & 31.2 & 37.1 & 32.0 \\
NYC & 36.3 & 36.9 & 27.9 & 37.8 \\
Football & 26.3 & 27.4 & 40.4 & 41.3 \\
Susie & 39.3 & 40.8 & 44.3 & 44.8 \\
Mother \& daughter & 44.0 & 44.7 & & \\
\hline
\end{tabular}




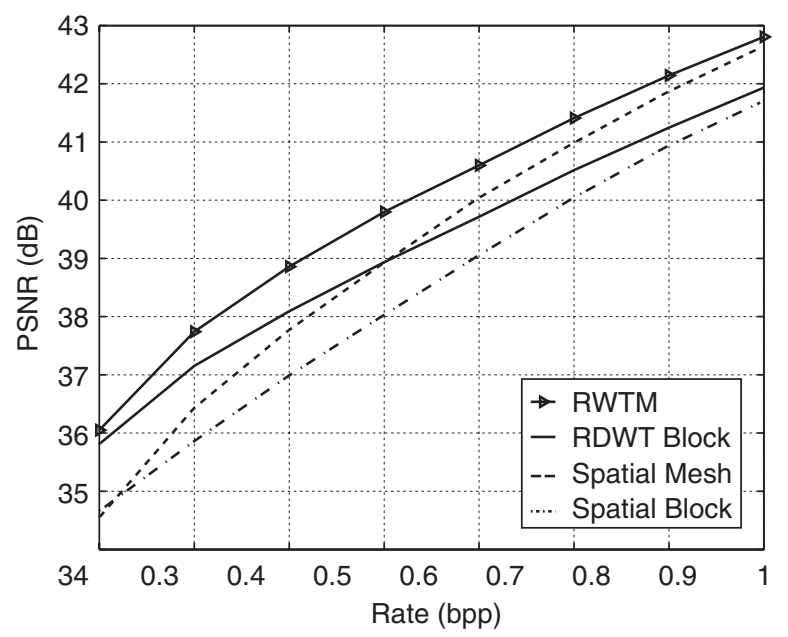

Fig. 8. Rate-distortion performance for "Foreman."

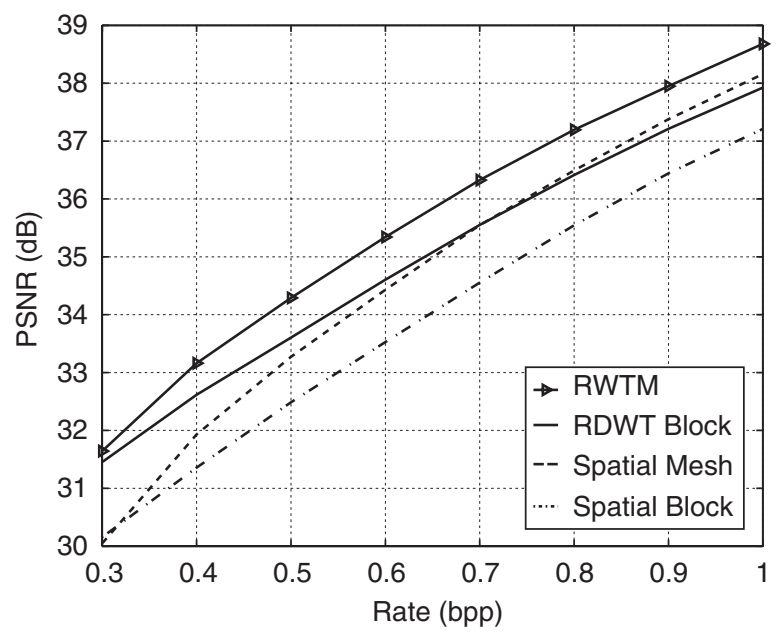

Fig. 9. Rate-distortion performance for "Table-tennis."

techniques for the slowly moving "Mother \& Daughter," and "Susie" sequences. We note that our combination of triangle-mesh $\mathrm{ME} / \mathrm{MC}$ and RDWT-based ME/MC outperforms either technique applied alone.

In terms of the computational complexity of the proposed RWTM technique, let us observe that, since the RDWT is significantly more computationally complex than its critically sampled counterpart, RWTM, which features two RDWT calculations as shown in Fig. 5, is a good deal slower than RDWT Block, which uses only one. RDWT Block, in turn, is significantly more complex than both of the spatial-domain approaches considered. Specifically,

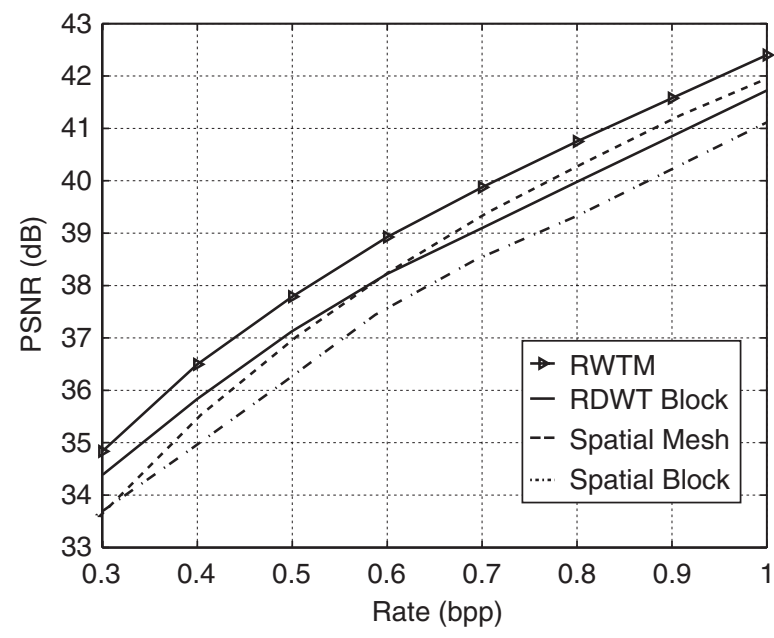

Fig. 10. Rate-distortion performance for "NYC."

in our experimental results, we have seen that RWTM has execution times roughly twice that of RDWT Block which in turn runs some six times slower than the spatial-domain approaches, although we must temper this observation with the caveat that none of our implementations have been optimized in any way for speed. We note that the computational burden of the RDWTs in RWTM can be greatly alleviated by resorting to an implementation of the transforms with fixed-point arithmetic, which, in turn, would permit fixed-point implementation of the MAE and affine-transformation operations described in Section 4. Finally, the cascade of the inverse DWT and forward RDWT in the ME/MC feedback loop of Fig. 5 can be significantly expedited by employing a direct complete-to-overcomplete transform [6,5,21]. As a consequence, a practical RWTM implementation is likely to be a good deal less computationally burdensome than our experimental observations might suggest.

\section{Conclusions}

The majority of prior approaches (e.g., $[25,20,23,24,4,3])$ to wavelet-domain $\mathrm{ME} / \mathrm{MC}$ have exploited merely the fact that the shift invariance of the RDWT makes it an ideal candidate for the implementation of $\mathrm{ME} / \mathrm{MC}$ in the wavelet domain. On the other hand, the RWTM technique that we present in this paper exploits the redundancy of the RDWT not only for its shift invariance, but also for the fact that it facilitates construction of a simple 


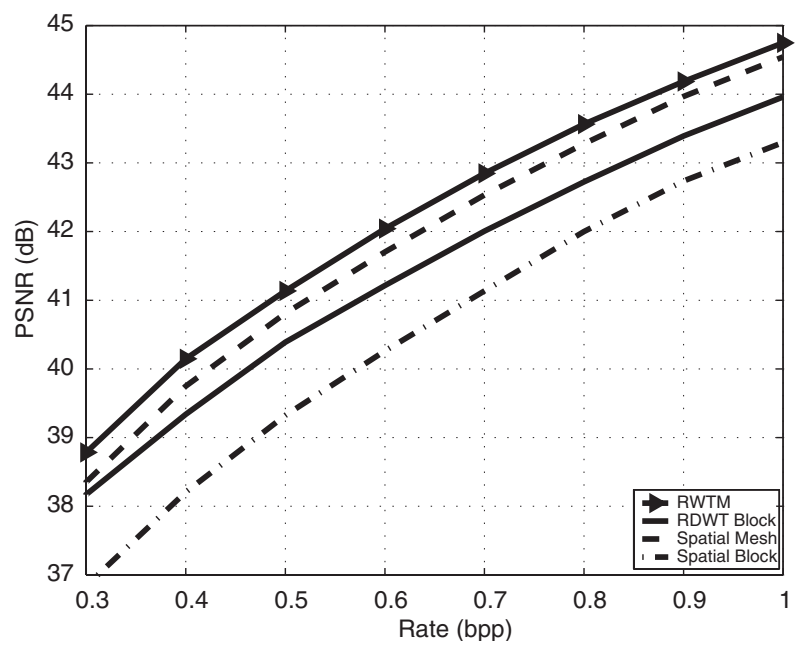

Fig. 11. Rate-distortion performance for "Susie."

correlation operator robust to noise. As we have argued, this correlation operator identifies salient image edges more accurately than gradient-based edge detectors operating in the spatial domain in the presence of noise. As a result, RWTM, which combines triangle-based meshes with RDWT-domain $\mathrm{ME} / \mathrm{MC}$, can more accurately anchor a triangle mesh to persistent edges, leading to ratedistortion performance superior to that of either an equivalent spatial-domain triangle-mesh approach or an RDWT-domain block-based approach. Empirical results indicate that this advantage is particularly pronounced when motion in the sequence is complex or fast moving.

\section{Acknowledgment}

This work was funded in part by the National Science Foundation under Grants CCR-0310864 and ACI-9982344.

\section{References}

[1] Y. Altunbasak, A.M. Tekalp, G. Bozdagi, Two-dimensional object-based coding using a content-based mesh and affine motion parameterization, in: Proceedings of the International Conference on Image Processing, vol. 2, Washington, DC, 1995, pp. 394-397.

[2] Y. Andreopoulos, A. Munteanu, J. Barbarien, M. van der Schaar, J. Cornelis, P. Schelkens, In-band motion compensated temporal filtering, Signal Process. Image Commun. 19 (2004) 653-673.

[3] Y. Andreopoulos, A. Munteanu, G. Van der Auwera, P. Schelkens, J. Cornelius, Wavelet-based fully-scalable video coding with in-band prediction, in: Proceedings of the Third IEEE Benelux Signal Processing Symposium, Leuven, Belgium, 2002, pp. 217-220.

[4] Y. Andreopoulos, A. Munteanu, G. Van der Auwera, P. Schelkens, J. Cornelius, Scalable wavelet video-coding with in-band prediction-implementation and experimental results, in: Proceedings of the International Conference on Image Processing, vol. 3, Rochester, NY, 2002, pp. 729-732.

[5] Y. Andreopoulos, A. Munteanu, G. Van der Auwera, P. Schelkens, J. Cornelius, A new method for complete-toovercomplete discrete wavelet transforms, in: Proceedings of the International Conference on Digital Signal Processing, Santorini, Greece, 2002, pp. 501-504.

[6] Y. Andreopoulos, A. Munteanu, G. Van der Auwera, J.P.H. Cornelius, P. Schelkens, Complete-to-overcomplete discrete wavelet transforms: theory and applications, IEEE Trans. Signal Process. 53 (4) (2005) 1398-1412.

[7] M. Antonini, M. Barlaud, P. Mathieu, I. Daubechies, Image coding using wavelet transform, IEEE Trans. Image Process. 1 (2) (1992) 205-220.

[8] V. Bhaskaran, K. Konstantinides, Image and Video Compression: Algorithms and Architectures, 2nd ed., Kluwer Academic Publishers, Boston, MA, 1997.

[9] R.J. Clarke, Digital Compression of Still Images and Video, Academic Press, London, 1995.

[10] S. Cui, Y. Wang, J.E. Fowler, Mesh-based motion estimation and compensation in the wavelet domain using a redundant transform, in: Proceedings of the International Conference on Image Processing, vol. 1, Rochester, NY, 2002, pp. 693-696.

[11] S. Cui, Y. Wang, J.E. Fowler, Multihypothesis motion compensation in the redundant wavelet domain, in: Proceedings of the International Conference on Image Processing, vol. 2, Barcelona, Spain, 2003, pp. 53-56.

[12] P. Dutilleux, An implementation of the "algorithme à trous" to compute the wavelet transform, in: J.-M. Combes, A. Grossman, P. Tchamitchian (Eds.), Wavelets: TimeFrequency Methods and Phase Space, Springer, Berlin, 
Germany, 1989, pp. 298-304, Proceedings of the International Conference, Marseille, France, December 14-18, 1987.

[13] M. Eckert, D. Ruiz, J.I. Ronda, N. Garcia, Evaluation of DWT and DCT for irregular mesh-based motion compensation in predictive video coding, in: K.N. Ngan, T. Sikora, M.-T. Sun (Eds.), Visual Communications and Image Processing, Proceedings of the SPIE, vol. 4067, 2000, pp. 447-456.

[14] J.E. Fowler, QccPack: an open-source software library for quantization, compression, and coding, in: A.G. Tescher (Ed.), Applications of Digital Image Processing XXIII, Proceedings of the SPIE, vol. 4115, San Diego, CA, 2000, pp. 294-301.

[15] J.E. Fowler, S. Cui, Y. Wang, Motion compensation via redundant-wavelet multihypothesis, IEEE Trans. Image Process., to appear.

[16] B. Girod, The efficiency of motion-compensating prediction for hybrid coding of video sequences, IEEE J. Selected Areas Commun. 5 (7) (1987) 1140-1154.

[17] M. Holschneider, R. Kronland-Martinet, J. Morlet, P. Tchamitchian, A real-time algorithm for signal analysis with the help of the wavelet transform, in: J.-M. Combes, A. Grossman, P. Tchamitchian (Eds.), Wavelets: TimeFrequency Methods and Phase Space, Springer, Berlin, Germany, 1989, pp. 286-297, Proceedings of the International Conference, Marseille, France, December 14-18, 1987.

[18] ITU-T, Video Coding for Audiovisual Services at $p \times 64$ kbit/s, ITU-T Recommendation H.261, March 1993.

[19] A.K. Jain, Fundamentals of Digital Image Processing, Prentice-Hall, Englewood Cliffs, NJ, 1989.

[20] H.S. Kim, H.W. Park, Wavelet-based moving-picture coding using shift-invariant motion estimation in wavelet domain, Signal Process. Image Commun. 16 (7) (2001) 669-679.

[21] X. Li, New results of phase shifting in the wavelet space, IEEE Signal Process. Lett. 10 (7) (2003) 193-195.

[22] X. Li, Scalable video compression via overcomplete motion compensated wavelet coding, Signal Process. Image Commun. 19 (2004) 637-651.

[23] X. Li, L. Kerofsky, S. Lei, All-phase motion compensated prediction in the wavelet domain for high performance video coding, in: Proceedings of the International Conference on
Image Processing, vol. 3, Thessaloniki, Greece, 2001, pp. 538-541.

[24] X. Li, L. Kerofsky, High-performance resolution-scalable video coding via all-phase motion-compensated prediction of wavelet coefficients, in: C.-C.J. Kuo (Ed.), Visual Communications and Image Processing, Proceedings of the SPIE, vol. 4671, 2002, pp. 1080-1090.

[25] H.-W. Park, H.-S. Kim, Motion estimation using low-bandshift method for wavelet-based moving-picture coding, IEEE Trans. Image Process. 9 (4) (2000) 577-587.

[26] A. Said, W.A. Pearlman, A new, fast, and efficient image codec based on set partitioning in hierarchical trees, IEEE Trans. Circuits Systems Video Technol. 6 (3) (1996) 243-250.

[27] A. Secker, D. Taubman, Highly scalable video compression using a lifting-based $3 \mathrm{D}$ wavelet transform with deformable mesh motion compensation, in: Proceedings of the International Conference on Image Processing, vol. 3, Rochester, NY, 2002, pp. 749-752.

[28] A. Secker, D. Taubman, Lifting-based invertible motion adaptive transform (LIMAT) framework for highly scalable video compression, IEEE Trans. Image Process. 12 (12) (2003) 1530-1542.

[29] M.J. Shensa, The discrete wavelet transform: wedding the à trous and Mallat algorithms, IEEE Trans. Signal Process. 40 (10) (1992) 2464-2482

[30] Y. Wang, S. Cui, J.E. Fowler, 3D video coding using redundant-wavelet multihypothesis and motion-compensated temporal filtering, in: Proceedings of the International Conference on Image Processing, vol. 2, Barcelona, Spain, 2003, pp. 755-758.

[31] Y. Wang, S. Cui, J.E. Fowler, 3D video coding with redundant-wavelet multihypothesis, IEEE Trans. Circuits Systems Video Technol. 16 (2) (2006) 166-177.

[32] Y. Xu, J.B. Weaver, D. Healy Jr., J. Lu, Wavelet transform domain filters: a spatially selective noise filtration technique, IEEE Trans. Image Process. 3 (6) (1994) 747-758.

[33] J.C. Ye, M. van der Schaar, Fully scalable 3-D overcomplete wavelet video coding using adaptive motion compensated temporal filtering, in: T. Ebrahimi, T. Sikora (Eds.), Visual Communications and Image Processing, Proceedings of the SPIE, vol. 5150, Lugano, Switzerland, 2003, pp. 1169-1180. 